\title{
Virtual Reality Training of Myoelectric Prosthesis with the Use of Sensory Feedback and Serious Game Techniques
}

\author{
Reidner Cavalcante ${ }^{\dagger}$ \\ Faculty of Electrical Engineering \\ Federal University of Uberlândia \\ Uberlândia, Brazil \\ reidnersc@outlook.com
}

\author{
Alcimar Soares \\ Faculty of Electrical Engineering \\ Federal University of Uberlândia \\ Uberlândia, Brazil \\ alcimar@ufu.br
}

\author{
Edgard Lamounier \\ Faculty of Electrical Engineering \\ Federal University of Uberlândia \\ Uberlândia, Brazil \\ lamounier@ufu.br
}

\begin{abstract}
In this thesis, we propose a system that uses immersive Virtual Reality (iVR) and EMG signal processing (muscle activity) to provide a training environment for amputees who are supposed to use a myoelectric prosthesis. We also investigate the efficiency of learning how to control a virtual prosthesis with and without sensory feedback. Our results show that virtual training can be greatly improved when proper tactile feedback is provided, especially for myoelectric controlled prostheses.
\end{abstract}

\section{INDEX TERMS}

K.4.2. [Computers and Society]: Social Issues-Assistive technologies for persons with disabilities.

\section{KEYWORDS}

Sensory feedback, Immersive Virtual Reality, myoelectric prosthesis, prosthetic training

\section{Introduction}

In the past years, Virtual Reality (VR) has been considered a potential approach for prosthetic training [1]. However, most VRbased upper-limb prosthetic training systems, reported in the literature, are based on non-immersive environments [2]. In fact, in many initiatives, users only visualize a virtual prosthesis, at a distance, on a flat screen. Unfortunately, this lacks intuition for real life prosthetic control.

Furthermore, sole visual feedback drastically limits the immersive experience for the purpose of prosthesis training, which requires users to reach and manipulate different objects. It is important to provide users with the feeling of touch to enhance embodiment experience and potentially provide sensations usually present when training with real devices. Such feedback is especially crucial for myoelectric prostheses.

This thesis raises the argument that an immersive virtual environment equipped with proper sensory feedback can significantly lessen the above drawbacks. We hypothesize that such integration can provide the user with a high immersion level in the virtual space.

The central motivation of this research is based on the search for a solution that can help the training of amputees of prostheses, from the development of an immersive virtual environment equipped with sensory feedback, in order to significantly reduce the disadvantages mentioned above.

\section{Related Work}

Various technologies using Electromyographic (EMG) and neural signal [3] have been described in the literature for virtual prosthesis control. The combination of EMG and kinematic data has been proposed to improve prosthesis control and better correlate the user's arm movements to the virtual prosthesis's motion within the virtual environment [4]. However, this system only provides visual feedback and does not explore the benefits of iVR for prosthesis training.

In [5], the user visualizes the environment through a headmounted display (HMD). In other scenarios, the user interacts with a non-immersive environment [6], where movements are projected onto an on-screen avatar. Immersive virtual environments have systematically shown to maintain the illusion of presence, which, in turn, positively impacts attention and engagement. Nevertheless, none of the above systems were designed for myoelectric control of the virtual prostheses, nor do they provide sensory feedback.

Consequently, the need to explore and present a solution that, in addition to presenting an interface using iVR techniques, also provides proper sensory feedback to enhance users' experience while training to control upper-limb myoelectric prostheses is justified. This is part of a PhD thesis and is related to Immersive gaming/serious game.

\section{Proposed Solution}

To evaluate this proposal, an iVR system has been designed. The system's main components are: HTC Vive Pro, Vive Tracker, EMG controller (data acquisition and pattern recognition), vibrational 
armband, and a computer (Fig. 1). The user interacts with the virtual environment by moving and contracting the stump muscles. In case of nonamputee volunteers, forearm muscles are used.

The Vive Tracker, located in the arm, provides information so that the virtual prosthesis moves within the virtual environment as the user moves his/her arm. The EMG module (Fig. 1) processes the contractions of the stump (or forearm) muscles and sends setpoint values to the virtual environment to indicate how much the virtual prosthesis must open/close the hand. The vibrational armband, and the EMG sensors are also positioned on the user's forearm. Whenever the user grabs an object in the virtual environment (Fig. 2), vibration feedback is sent to the user to convey touch. If the object is dropped or released, the feedback stops.

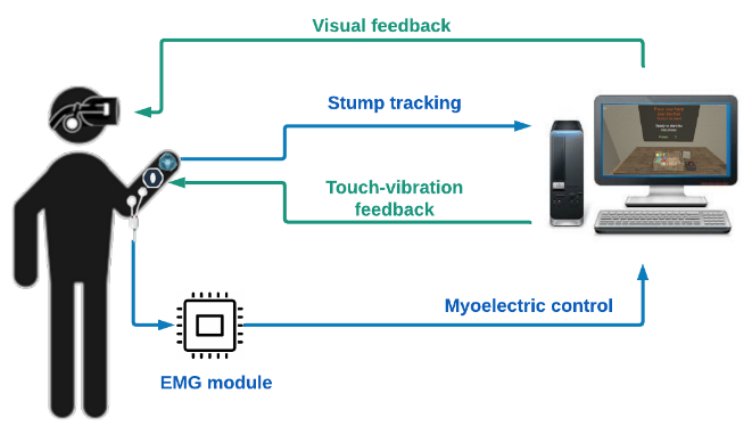

Figure 1: System Architecture.

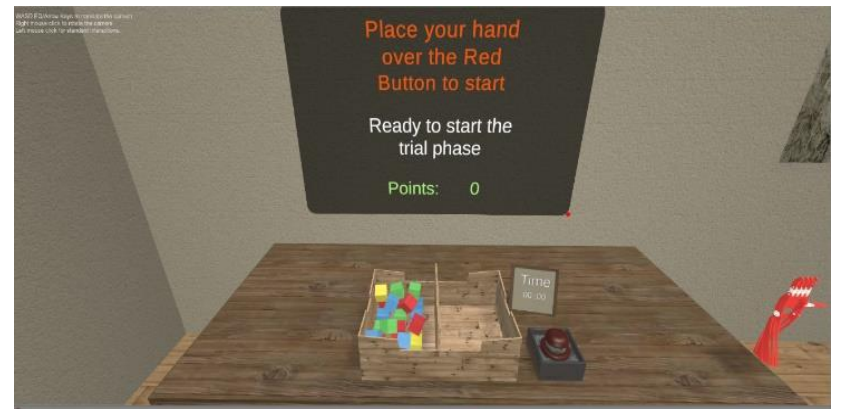

Figure 2: iVR environment for the Box and Blocks Test

\section{Preliminary Results}

For this study, eight non-amputee male volunteers, ages between 20 and 40, were recruited. These volunteers were divided into two groups: G1 - performed the B\&B tasks while receiving visual and vibrational tactile feedback; G2 - performed the B\&B tasks while receiving only visual feedback.

In the first sessions, the volunteers learn how to control the virtual prosthesis using myoelectric signals and adapting to the virtual environment. Accordingly, the first two trials required a longer time to complete. As the trials progressed, especially for the volunteers of G1, completion time decreased steadily. Completion time for the volunteers of G1 during Trial 1 vary from 17.67 to 209.97 seconds (mean 100.99 seconds); completion time for Trial 2 ranged from 25.39 to 82.32 seconds (mean 47,6 seconds); for Trial 3 from 17.1 to 62.22 seconds (mean 36,75 seconds); and for Trial 4 from 17.59 to 34.25 seconds (mean 22.21).
In contrast, the completion time for the volunteers of $\mathrm{G} 2$ during Trial 1 vary from 28.06 to 135.12 seconds (mean 66.25 seconds); completion time for Trial 2 ranged from 10.4 to 207.38 seconds (mean 85.77 seconds); for Trial 3 from 11.81 to 42.67 seconds (mean 32.49 seconds); and for Trial 4 from 13.94 to 96.36 seconds (mean 50.87).

Although the initial mean value for G1 was considerably higher than the initial mean value for G2 (mainly due to the very high time taken by G1-V3 to complete the first trial), the group using vibrational tactile feedback showed a better performance overall. On average G2 took 2.3 times longer to complete the task at the fourth trial.

\section{Conclusion}

In this paper, we investigated the importance of sensory feedback to enhance embodiment during myoelectric prostheses using iVR. We developed an immersive virtual environment where the users can perform the tasks required by Box \& Blocks Test using a virtual myoelectric prosthesis and vibration feedback.

Although both experimental groups showed improvement in the control of the myoelectric virtual prosthesis over the trials, participants of the group using tactile vibrational feedback showed better performance as the trials progressed, compared with the group that did not use tactile feedback. Those results indicate that sensory feedback is indeed capable of providing a better overall experience while optimizing training sessions using immersive virtual reality environments.

As soon as the current COVID-19 pandemic crises subsides, future steps for this work involve extensive tests with amputee subjects seeking to perfect the system with that population.

\section{ACKNOWLEDGMENTS}

This work was supported in part by the Coordination for the Improvement of Higher Education Personnel - Brazil (CAPES) and Qatar University under the grant IRCC-2019-001. The statements made herein are solely the responsibility of the authors.

\section{REFERENCES}

[1] J. M. Churko, A. Mehr, A. G. Linassi, and A. Dinh, "Sensor evaluation for tracking upper extremity prosthesis movements in a virtual environment," in Proceedings of the 31st Annual International Conference of the IEEE Engineering in Medicine and Biology Society: Engineering the Future of Biomedicine, EMBC 2009, 2009, vol. 2009, pp. 2392-2395..

[2] M. Melero et al., "Upbeat: Augmented Reality-Guided Dancing for Prosthetic Rehabilitation of Upper Limb Amputees," J. Healthc. Eng., vol. 2019, 2019.

[3] K. Li, P. Boyd, Y. Zhou, Z. Ju, and H. Liu, "Electrotactile Feedback in a Virtual Hand Rehabilitation Platform: Evaluation and Implementation," IEEE Trans. Autom. Sci. Eng., vol. 16, no. 4, pp. 1556-1565, Oct. 2019.

[4] D. Blana, T. Kyriacou, J. M. Lambrecht, and E. K. Chadwick, "Feasibility of using combined EMG and kinematic signals for prosthesis control: A simulation study using a virtual reality environment," J. Electromyogr. Kinesiol., vol. 29, pp. 21-27, Aug. 2016.

[5] K. Odette and Q. Fu, "A Physics-based Virtual Reality Environment to Quantify Functional Performance of Upper-limb Prostheses," in Proceedings of the Annual International Conference of the IEEE Engineering in Medicine and Biology Society, EMBS, 2019, vol. 2019, pp. 3807-3810.

[6] T. Shibanoki, G. Nakamura, T. Tsuji, K. Hashimoto, and T. Chin, "A New Approach for Training on EMG-based Prosthetic Hand Control," in LifeTech 2020 - 2020 IEEE 2nd Global Conference on Life Sciences and Technologies, 2020, pp. 307-308. 